\title{
Ribaucour coordinates
}

\author{
F. Burstall ${ }^{1}(1) \cdot$ U. Hertrich-Jeromin ${ }^{2}([)$ \\ M. Lara Miro ${ }^{2}$
}

\begin{abstract}
We discuss results for the Ribaucour transformation of curves or of higher dimensional smooth and discrete submanifolds. In particular, a result for the reduction of the ambient dimension of a submanifold is proved and the notion of Ribaucour coordinates is derived using a Bianchi permutability theorem. Further, we discuss smoothing of semi-discrete curvature line nets and an interpolation by Ribaucour transformations.
\end{abstract}

Keywords Channel surface Canal surface $\cdot$ Semi-discrete surface $\cdot$ Curvature line net - Discrete principal net - Circular net - Ribaucour transformation - Ribaucour coordinates

Mathematics Subject Classification 53C42 - 53A10 - 53A30 - 37K25 · 37K35

\section{Introduction}

This paper touches upon several ideas and results concerning the Ribaucour transformation of submanifolds and, in particular, of curves: the 1-dimensional case not only helps to illustrate the main ideas of our investigations, but it is also of interest for the construction of 2-dimensional discrete and semi-discrete principal "circular" nets and

\footnotetext{
$凶$ U. Hertrich-Jeromin

udo.hertrich-jeromin@tuwien.ac.at

F. Burstall

feb@maths.bath.ac.uk

M. Lara Miro

mmiro@geometrie.tuwien.ac.at

1 Department of Mathematical Sciences, University of Bath, Bath BA2 7AY, UK

2 Vienna University of Technology, Wiedner Hauptstraße 8-10/104, 1040 Vienna, Austria
} 
for their "smoothing", cf Burstall et al. (2016). On the other hand, our discussion of higher dimensional submanifolds not only provides a generalization of some of the results for curves, but it also sheds light on the reasons and structure behind the results for curves. Thus we first present several results for the Ribaucour transformation of curves and then discuss whether and how these generalize to higher dimensions: a reduction of the ambient dimension by means of Ribaucour transformation, which leads to Ribaucour coordinates; smoothing of a sequence of Ribaucour transforms; and the possibility of an interpolation between curves or submanifolds by means of a sequence of Ribaucour transformations. Each of these problems is addressed in both the smooth and discrete settings.

Ribaucour coordinates were used by the classical authors to investigate the geometry of surfaces with particular properties of their curvature lines: given a surface and a suitable plane, for example, a tangent plane of the surface, one may locally construct a regular map of the surface to the plane by means of touching 2 -spheres. These "Ribaucour coordinates" then map the curvature line net of the surface to an orthogonal net in the plane. For example, it is advantageous to employ this type of coordinates to investigate and construct surfaces of constant mean curvature $H=1$ in hyperbolic space, cf de Lima and Roitman (2002) or Hertrich-Jeromin (2003, §5.5.27). These classical Ribaucour coordinates were generalized in more recent work to hypersurfaces, see Corro and Tenenblat (2004, Cor 2.10), and to submanifolds with flat normal bundle, see Dajczer et al. (2007, Thm 1).

One principal aim of this paper is to provide a more direct geometric approach to these generalized Ribaucour coordinates, much in the spirit of the classical authors. This approach relies on a geometric method to reduce the ambient dimension of a curve or submanifold by means of a Ribaucour transformation into a hypersphere, see Corollary 2.5, Theorem 2.6 for (smooth and discrete) curves and Corollary 3.6, Corollary 3.7 for submanifolds. Once the dimension reduction is established, the higher codimension version of Ribaucour coordinates relies on a Bianchi permutability result, cf Dajczer et al. (2007, Thm 2) and Hertrich-Jeromin (2003, §8.5.8). In our setting, this permutability result is readily verified by elementary means, leading to Ribaucour coordinates for any smooth submanifold with flat normal bundle resp any discrete circular net of higher codimension, see Theorems 3.8 and 3.9.

Another observation that relies on rather similar ideas leads to a "smoothing" of a semi-discrete curvature line net, since such a net can be thought of as a sequence of Ribaucour transforms of one curve. If two $m$-dimensional submanifolds envelop an $m$-sphere congruence then this sphere congruence provides a metric and connection preserving isomorphism field between normal bundles, see Lemmas 2.1 and 3.4, cf Corro and Tenenblat (2004, Cor 2.9). As a consequence, any pair of curves that envelop a 1-parameter family of circles, that is, form a Ribaucour pair, are two curvature lines of a channel surface, see Corollary 2.4 or Fig. 3: this result can be thought of as the semi-discrete version of a "smoothing" result for discrete circular nets, see Bobenko and Huhnen-Venedy (2012) and Bo et al. (2011), where a discrete circular net is "smoothed" by fitting Dupin cyclide patches into its facets. The higher dimensional version Corollary 3.5 emphasizes that the construction only depends on an enveloped $m$-sphere congruence, not the fact that the two submanifolds form a Ribaucour pair: if they do, more structure can be obtained. 

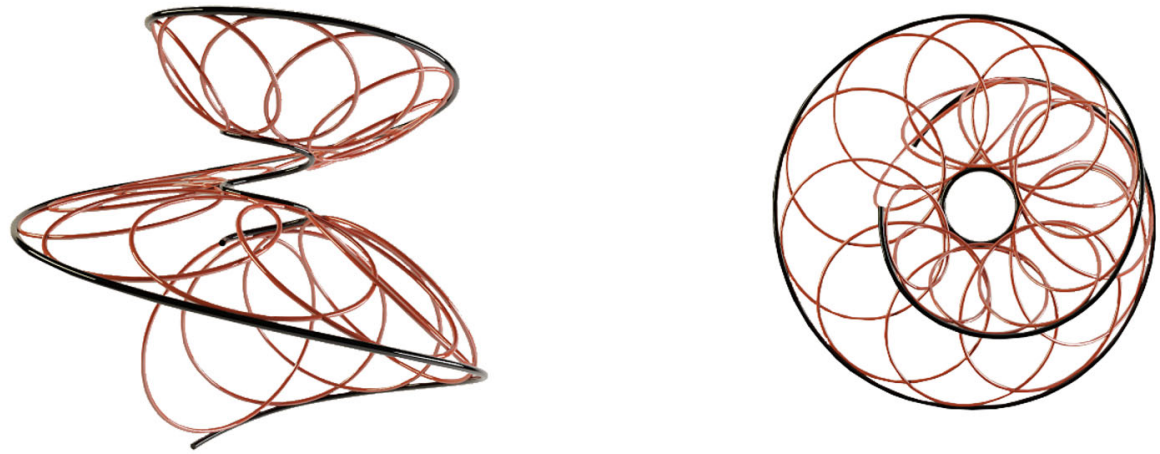

Fig. 1 A Ribaucour pair of curves

Finally, we prove that any two (smooth or discrete) curves can be transformed into each other by a sequence of Ribaucour transforms, see Corollary 2.9. In contrast to Cauchy problems for discrete nets, cf Bobenko and Hertrich-Jeromin (2001, Sect 4) or Hertrich-Jeromin (2003, §8.4.9), this is a mixed boundary and initial value problem and allows to construct discrete or semi-discrete principal nets with given boundary data. This result hinges again on the aforementioned dimension reduction, which allows us to reduce the problem to planar curves. For planar curves, the result is then proved by a simple geometric construction in the discrete case, and as a trivial consequence of Burstall and Hertrich-Jeromin (2006, Thm 3.4) in the smooth case, see Theorem 2.8. However, as the flatness of a certain vector bundle is required-which is trivial in the 1-dimensional case, but not in higher dimensions-our interpolation result does not generalize to higher dimensions.

Though we start by formulating our results for curves in Euclidean space in order to make the text more accessible, we quickly resort to sphere geometric methods: as the Ribaucour transformation is a Lie geometric notion, many arguments are more efficient and transparent in the Möbius or Lie geometric settings. Since these techniques are discussed in great detail in other works, we will only give few hints or details where required, and refer the reader to, for example, Hertrich-Jeromin (2003) as well as to our previous papers Burstall and Hertrich-Jeromin (2006) or Burstall et al. (2016).

\section{Space curves}

To set the scene we discuss smooth and discrete curves in 3-space: this case already displays most of the key ideas of this study, without the additional complexity caused by the partial differential equations occurring in the case of higher dimensional submanifolds.

Thus let $x, \hat{x}: I \rightarrow \mathbb{R}^{3}$ denote a Ribaucour pair of curves, that is, $x$ and $\hat{x}$ share a common tangent circle $c(u)$ at corresponding points $x(u)$ and $\hat{x}(u)$, cf Burstall et al. (2016, Def 2.1) and Fig. 1. We say that the two curves are mutual Ribaucour transforms of each other. For regularity we assume $x(u) \neq \hat{x}(u)$ for all $u \in I$. Analytically, this relation between the two curves can be encoded by the reality of their tangent cross ratio, 


$$
c r:=x^{\prime}(\hat{x}-x)^{-1} \hat{x}^{\prime}(\hat{x}-x)^{-1}: I \rightarrow \mathbb{R},
$$

where products and inverses of vectors $v \in \mathbb{R}^{3} \cong \operatorname{Im} \mathbb{H}$ are computed in terms of Clifford, or quaternionic, multiplication, see Burstall et al. (2016, Lemma 2.2). This (quadratic) relation between the two curves may be linearized by means of standard Möbius geometric methods, see Burstall et al. (2016, Lemma 2.3): replacing $\mathbb{R}^{3}$ by the conformal 3-sphere, thought of as the projective light cone in $\mathbb{R}^{4,1}$,

$$
S^{3} \cong \mathbb{P}\left(\mathcal{L}^{4}\right) \text { with } \mathcal{L}^{4}=\left\{y \in \mathbb{R}^{4,1} \mid(y, y)=0\right\},
$$

the circularity condition becomes linear in terms of homogeneous coordinates $x$ and $\hat{x}$. In detail, consider the orthogonal decomposition

$$
\mathbb{R}^{4,1}=\mathbb{R}^{1,1} \oplus_{\perp} \mathbb{R}^{3}, \text { where } \mathbb{R}^{1,1}=\langle o, q\rangle
$$

is spanned by isotropic vectors $o, q \in \mathbb{R}^{4,1}$ with inner product $(o, q)=-1$, and the Euclidean lift of a curve in $\mathbb{R}^{3}$ into the light cone obtained from the isometric embedding

$$
\mathbb{R}^{3} \ni x \mapsto \xi:=o+x+\frac{1}{2}(x, x) q \in \mathcal{Q}^{3}:=\left\{y \in \mathcal{L}^{4} \mid(y, q)=-1\right\} \subset \mathcal{L}^{4}
$$

The circularity condition of the Ribaucour transformation for two curves now reduces to linear dependence: $u \mapsto \operatorname{dim}\left\langle\xi, \xi^{\prime}, \hat{\xi}, \hat{\xi}^{\prime}\right\rangle(u)=3$. Clearly, this condition is independent of the scaling of lifts $\xi \in \Gamma\left(\left\langle o+x+\frac{1}{2}(x, x) q\right\rangle\right)$ of $x$ and $\hat{\xi}$ of $\hat{x}$, in fact, the map

$$
u \mapsto c(u):=\left\langle\xi, \xi^{\prime}, \hat{\xi}, \hat{\xi}^{\prime}\right\rangle(u)
$$

is independent of lifts and, in the case of a Ribaucour pair, encodes the enveloped circle congruence as a bundle of (projective light cones in) $(2,1)$-planes in $\mathbb{R}^{4,1}$, cf Hertrich-Jeromin (2003, §6.4.12 and §6.6.5). Similarly, a 2-sphere can be encoded by a $(3,1)$-plane in $\mathbb{R}^{4,1}$ or, equivalently, by its orthogonal complement: normalizing yields an identification of hyperspheres with points in the Lorentz sphere

$$
S^{3,1}=\left\{y \in \mathbb{R}^{4,1} \mid(y, y)=1\right\},
$$

where a choice of sign can be used to encode orientation of a hypersphere. In particular we obtain representatives in $S^{3,1}$ for a sphere with centre $m$ and radius $r$ or a plane with unit normal $n$ through $x$ in $\mathbb{R}^{3}$ by (cf Hertrich-Jeromin 2003, Sect 1.1)

$$
s=\frac{1}{r}\left(o+m+\frac{(m, m)-r^{2}}{2} q\right) \operatorname{resp} t=n+(n, x) q .
$$



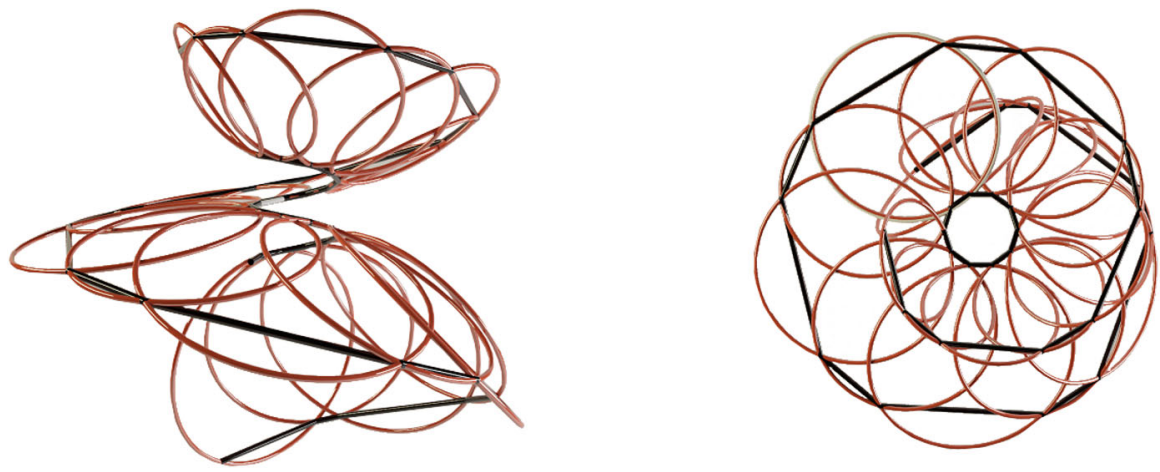

Fig. 2 A Ribaucour pair of discrete curves

Note that the above definition of Ribaucour pairs of curves resonates well with the discrete case, cf Bobenko and Hertrich-Jeromin (2001, Sect 4) or Hertrich-Jeromin (2003, §8.3.16), and Fig. 2: two discrete curves $x, \hat{x}: \mathbb{Z} \supset I \rightarrow \mathbb{R}^{3}$ are said to form a Ribaucour pair if endpoints of corresponding edges are concircular, that is, for adjacent $i, j \in I$,

$$
c_{i j}:=\left\langle\xi_{i}, \xi_{j}, \hat{\xi}_{i}, \hat{\xi}_{j}\right\rangle \cong \mathbb{R}^{2,1} \subset \mathbb{R}^{4,1}
$$

Now let $n: I \rightarrow S^{2}$ denote a unit normal field along one curve $x: I \rightarrow \mathbb{R}^{3}$ of a Ribaucour pair: this uniquely defines a congruence of hyperspheres $s(u)$ that contain corresponding points $x(u)$ and $\hat{x}(u)$ of both curves and have $n(u)$ as a normal; aligning orientations this construction yields an isometric isomorphism of normal bundles for the curves of a Ribaucour pair. Indeed, employing Möbius geometric lifts

$$
t=n+(n, x) q \in S^{3,1}
$$

of unit normals, the corresponding (hyper-)spheres can be encoded as a map into the Lorentz sphere,

$$
s=t+\frac{1}{r} \xi: I \rightarrow S^{3,1} \quad \text { with } \frac{1}{r}=-\frac{(\hat{\xi}, t)}{(\hat{\xi}, \xi)}
$$

and the induced normal field $\hat{n}$ of $\hat{x}$ is hence given by

$$
\hat{t}=s-\frac{1}{r} \hat{\xi}=t+\frac{1}{r}(\xi-\hat{\xi}): I \rightarrow S^{3,1}
$$

Lemma 2.1 Suppose $x, \hat{x}: I \rightarrow \mathbb{R}^{3}$ form a Ribaucour pair of curves; then the isomorphism (2.7) of normal bundles maps parallel normal fields $n$ of $x$ to parallel normal fields $\hat{n}$ of $\hat{x}$, that is, (2.7) intertwines normal connections of the two curves.

To substantiate this claim first observe that a normal field $n$ is parallel if and only if its lift (2.5) is, 


$$
n^{\prime}\left\|x^{\prime} \Leftrightarrow t^{\prime}\right\| \xi^{\prime}
$$

hence if and only if any sphere congruence

$$
s=t+\frac{1}{r} \xi \text { satisfies } s^{\prime} \in \Gamma\left(\left\langle\xi, \xi^{\prime}\right\rangle\right) .
$$

On the other hand, the enveloped sphere congruence (2.6) satisfies

$$
s=t+\frac{1}{r} \xi=\hat{t}+\frac{1}{r} \hat{\xi} \perp c=\left\langle\xi, \xi^{\prime}, \hat{\xi}, \hat{\xi}^{\prime}\right\rangle
$$

consequently, $s^{\prime} \perp \xi, \hat{\xi}$ so that the claim follows: $s^{\prime} \in \Gamma\left(\left\langle\xi, \xi^{\prime}\right\rangle\right)$ if and only if $s^{\prime} \in \Gamma\left(\left\langle\hat{\xi}, \hat{\xi}^{\prime}\right\rangle\right)$.

On the other hand, reversing this line of thought, we are able to reconstruct the enveloped circle congruence of a Ribaucour pair from a suitable enveloped (hyper-)sphere congruence: suppose that a sphere congruence $s: I \rightarrow S^{3,1}$ touches two curves $x$ and $\hat{x}$ and yields parallel normal fields for both curves,

$$
s \perp \xi, \xi^{\prime}, \hat{\xi}, \hat{\xi}^{\prime} \text { and } s^{\prime} \in \Gamma\left(\left\langle\xi, \xi^{\prime}\right\rangle\right) \cap \Gamma\left(\left\langle\hat{\xi}, \hat{\xi}^{\prime}\right\rangle\right)
$$

as $s^{\prime} \perp \xi, \hat{\xi}$ we conclude that

$$
\left.\begin{array}{l}
\xi^{\prime} \in \Gamma\left(\left\langle\xi, s^{\prime}\right\rangle\right) \\
\hat{\xi}^{\prime} \in \Gamma\left(\left\langle\hat{\xi}, s^{\prime}\right\rangle\right)
\end{array}\right\} \Rightarrow\left\langle\xi, \xi^{\prime}, \hat{\xi}, \hat{\xi}^{\prime}\right\rangle=\left\langle\xi, \hat{\xi}, s^{\prime}\right\rangle .
$$

Thus we have proved:

Lemma 2.2 Suppose that a curve $s: I \rightarrow S^{3,1}$ of spheres touches two curves $x, \hat{x}$ : $I \rightarrow \mathbb{R}^{3}$ and yields parallel normal fields for these curves; then $x$ and $\hat{x}$ form $a$ Ribaucour pair of curves.

As a curve on a surface is a curvature line if and only if the Gauss map of the surface yields a parallel normal field along the curve we deduce the following theorem for the channel surface enveloping one of the curves of spheres discussed above, cf Pember and Szewieczek (2017, Sect 5):

Corollary 2.3 Any two (non-circular) curvature lines of a channel surface form a Ribaucour pair of curves; given a Ribaucour pair of curves $x, \hat{x}: I \rightarrow \mathbb{R}^{3}$ there is a 1-parameter family of channel surfaces that contain both curves as curvature lines.

The first claim follows directly from Lemma 2.2, as the 1-parameter family of spheres enveloped by a channel surface yields parallel normal fields along any two of its (non-circular) curvature lines; the second claim follows from Lemma 2.1, by using one of the sphere congruences (2.6) given by a parallel normal field $n$ along $x$ to obtain one of the sought-after channel surfaces.

Recall that a semi-discrete curvature line net can be thought of as a sequence of curves, where subsequent curves form Ribaucour pairs, cf Müller and Wallner (2013, 
Fig. 3 A semi-discrete curvature line net, "smoothed" by channel surfaces

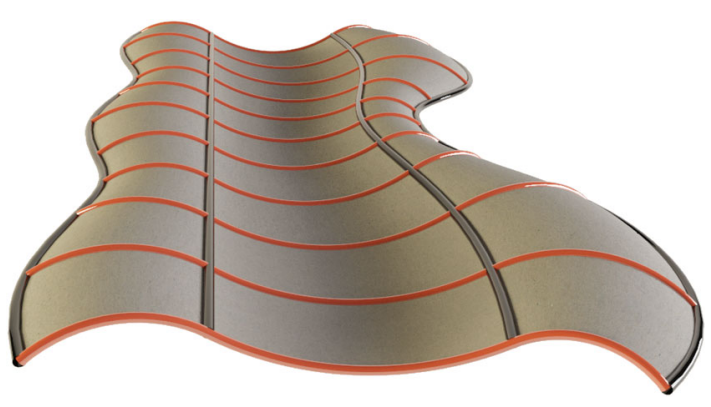

Def 1.1) or Burstall et al. (2016, Sect 3). As the construction of Corollary 2.3 of a channel surface from a Ribaucour pair of curves involves the choice of a parallel normal field along one of the two curves, this construction may be iterated to "smoothen" a semi-discrete curvature line net, see Fig. 3. reminiscent of the smoothing of fully discrete curvature line nets by using Dupin cyclides, cf Bobenko and Huhnen-Venedy (2012):

Corollary 2.4 Any semi-discrete curvature line net can be "smoothed" by a sequence of channel surfaces: it lies on a $C^{1}$-surface composed of channel surfaces that meet at the curves of the semi-discrete net and have the same tangent planes there.

This relation between Ribaucour pairs of curves and enveloped (hyper-)sphere congruences also yields an approach to reduce the ambient dimension of a curve by means of a Ribaucour transformation: given a curve $x: I \rightarrow \mathbb{R}^{3}$ we may use a parallel normal field $n: I \rightarrow S^{2}$ to construct a sphere congruence touching a given, fixed sphere and thereby producing a Ribaucour transform of $x$ that is contained in this sphere - as the radial vector field of a fixed sphere is parallel along any curve in that sphere the claim is an immediate consequence of Lemma 2.2.

Using Möbius geometric techniques again, the enveloped 1-parameter family of curves and the sought-after Ribaucour transform $\hat{x}$ of $x$ can be determined explicitly, by algebra alone: if

$$
\xi: I \rightarrow \mathcal{L}^{4} \text { and } t: I \rightarrow S^{3,1}
$$

denote (Möbius geometric) lifts of the curve $x$ and a parallel normal field $n$ along $x$, then

$$
s=t+\frac{1}{r} \xi
$$

defines a sphere curve that touches the curve, $s \perp \xi$, $\xi^{\prime}$, as well as a given fixed sphere $e \in S^{3,1}$ when we let $\frac{1}{r}:=\frac{1-(e, t)}{(e, \xi)}$ so that $(s, e) \equiv 1$;

$$
\hat{\xi}:=s-e: I \rightarrow \mathcal{L}^{4}
$$



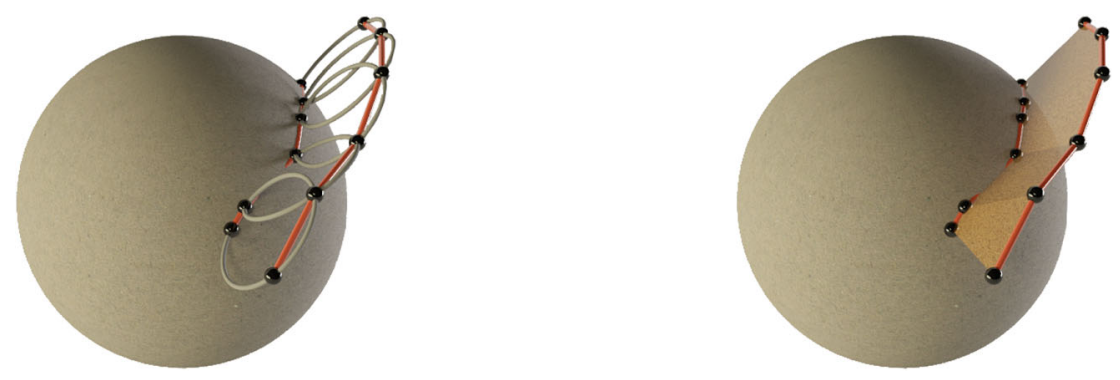

Fig. 4 Ambient dimension reduction by means of Ribaucour transformation

then yields (a light cone lift of) the touching point of $s$ and $e$, that is, of the desired Ribaucour transform $\hat{x}$ of $x$.

Corollary 2.5 Given a (fixed) sphere, any curve $x: I \rightarrow \mathbb{R}^{3}$ that does not meet the sphere can be Ribaucour transformed into the sphere by means of a parallel normal field $n$ along $x$; once a parallel normal field is given, the construction of the transform is algebraic.

The first part of Corollary 2.5 works in a completely analogous way for discrete curves: given a sphere that the curve does not meet, a Ribaucour transform of the curve can be constructed by iteratively constructing second intersection points of the sphere with the circumcircles of the endpoints of an edge and an initial point for the edge on the sphere, cf Fig. 4:

Theorem 2.6 Given a sphere, any discrete curve $x: \mathbb{Z} \supset I \rightarrow \mathbb{R}^{3}$ that does not meet the sphere can be Ribaucour transformed onto the sphere; this construction depends on the choice of one initial point on the sphere.

At no point of the argument did we use that the ambient dimension of the curve $x$ be 3, hence Corollary 2.5 and Theorem 2.6 hold in any dimension-in particular, also for planar curves: this leads to Ribaucour coordinates for a given curve, similar to the Ribaucour coordinates used by the classical geometers for surfaces, $\mathrm{cf}$ de Lima and Roitman (2002), and generalized for submanifolds in Dajczer et al. (2007, Thm 1). Namely, iterating the dimension reduction of Corollary 2.5 (or Theorem 2.6) we obtain a circular arc as a double Ribaucour transform of a space curve $x: I \rightarrow \mathbb{R}^{3}$. Suitably aligning iterated transformations, see Theorems 3.8 and 3.9, the order of transformations is irrelevant and we obtain Ribaucour coordinates for the given curve:

Corollary and Definition 2.7 Any curve $x: I \rightarrow \mathbb{R}^{3}$ is locally obtained by two subsequent commuting Ribaucour transforms from a circular arc;

the coordinates for the curve obtained in this way are called Ribaucour coordinates of the curve.

A similar line of thought can be used to interpolate between curves by Ribaucour transformations. In the discrete case the construction is straightforward: once the ambient dimension of the curves has been reduced to obtain a pair of spherical (or 

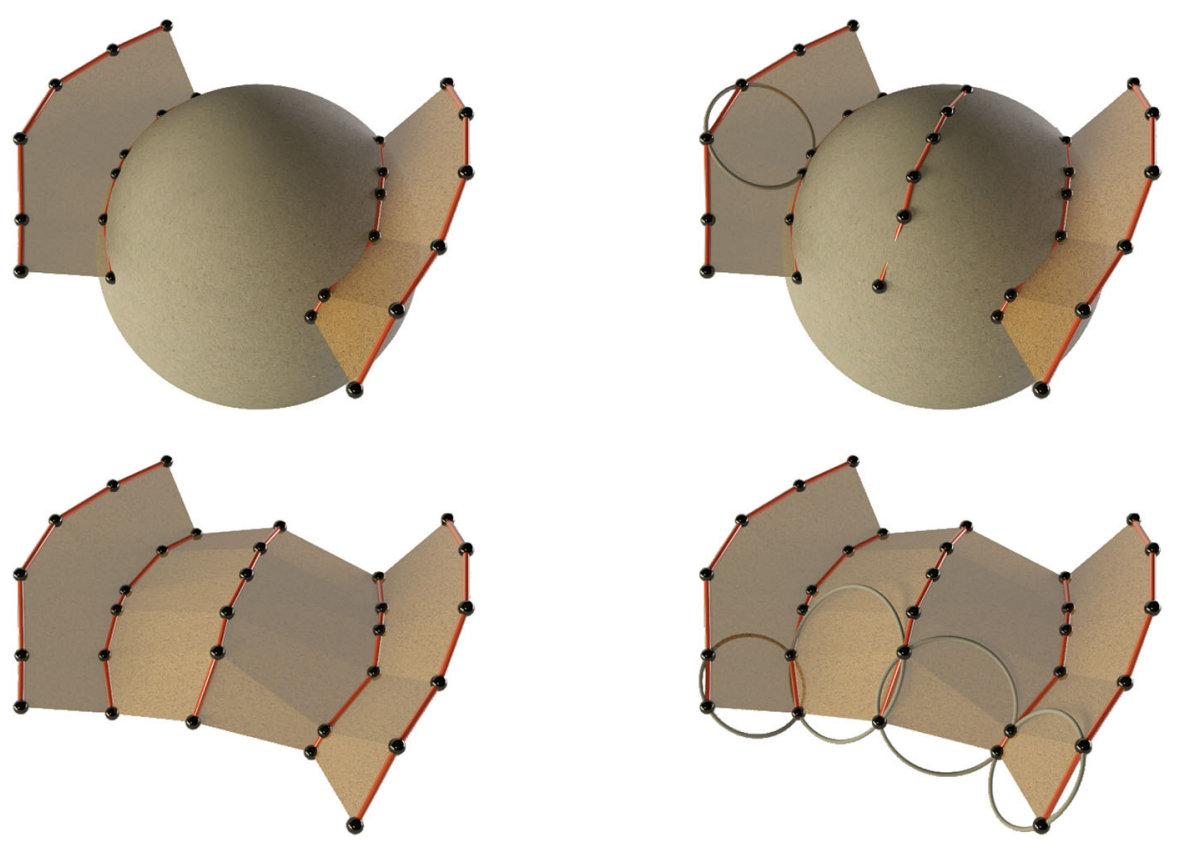

Fig. 5 Interpolation by Ribaucour transformations

planar) discrete curves, an "interpolating" Ribaucour transform of both curves may be constructed iteratively, by intersecting circumcircles of corresponding edges and a common initial point for the edges, cf Fig. 5.

In the smooth case the argument is similar: after a dimension reduction a common Ribaucour transform of two planar or spherical curves needs to be constructed. This is slightly more involved: a simple existence argument relies on Burstall and HertrichJeromin (2006, Thm 3.4), where we use methods from Lie sphere geometry. Thus we add another (timelike) dimension to the coordinate space of the Möbius geometric model,

$$
\mathbb{R}^{3,2}=\langle p\rangle \oplus_{\perp} \mathbb{R}^{3,1}=\langle o, p, q\rangle \oplus_{\perp} \mathbb{R}^{2}
$$

cf Blaschke (1929) or Burstall and Hertrich-Jeromin (2006); we then get a Legendre lift of a curve $x: I \rightarrow \mathbb{R}^{2}$ with unit normal field $n: I \rightarrow S^{1}$ by

$$
\lambda:=\langle\xi, v\rangle \text { with } v:=p+t=p+n+(n, x) q,
$$

that is, a curve of null 2-planes in $\mathbb{R}^{3,2}$, resp lines in the Lie quadric $\mathbb{P}\left(\mathcal{L}^{4}\right) \subset \mathbb{P}\left(\mathbb{R}^{3,2}\right)$, that satisfies the contact condition,

$$
\sigma^{\prime} \perp \lambda \text { for } \sigma \in \Gamma(\lambda)
$$


Now suppose that two curves $x_{i}: I \rightarrow \mathbb{R}^{2}(i=0,1)$ with unit normal fields $n_{i}$ are given and assume, for regularity, that the two curves do not touch a common circle at any two corresponding points $x_{i}(u)$. Then

$$
b:=\lambda_{0} \oplus \lambda_{1}: I \rightarrow G_{2,2}\left(\mathbb{R}^{3,2}\right)
$$

defines a $(2,2)$-bundle as a map into the Grassmannian of $(2,2)$-planes in $\mathbb{R}^{3,2}$. As this is a bundle over a 1-dimensional base it is clearly a flat bundle, thus Burstall and Hertrich-Jeromin (2006, Thm 3.4) assures that any null 2-plane subbundle $\hat{\lambda}$ of $b$ that intersects $\lambda_{i}$ non-trivially defines a common Ribaucour transform $\hat{x}: I \rightarrow \mathbb{R}^{2}$ of the two initial curves $x_{i}$.

Thus we obtain the following theorem that holds for both, smooth as well as discrete curves:

Theorem 2.8 Any two planar curves $x_{0}, x_{1}: I \rightarrow \mathbb{R}^{2}$ admit, under mild regularity assumptions, a common Ribaucour transform $\hat{x}: I \rightarrow \mathbb{R}^{2}$; in the smooth and discrete cases there is a 1-resp 2-parameter family of such common Ribaucour transforms.

As a consequence any two (discrete or smooth) space curves span a discrete resp semi-discrete curvature line net: we can solve a mixed boundary and initial value problem for (semi-)discrete curvature line nets. More precisely:

Corollary 2.9 Any two discrete or smooth space curves $x_{0}, x_{1}: I \rightarrow \mathbb{R}^{3}$ form two boundary curves of a discrete resp semi-discrete curvature line net; generically, three interpolating Ribaucour transforms are required.

\section{Submanifolds}

After putting forward the key ideas of this work in the previous section we are now prepared to tackle the general case of submanifolds in $\mathbb{R}^{n}$ or $S^{n}$. In the case of space curves, we based our presentation on two lemmas to then easily deduce the results we were interested in-however, while Lemma 2.1 is insensitive to dimensions, and a useful reformulation will be readily available, Lemma 2.2 crucially depends on the "correct" dimensions, and we will therefore need to use different arguments to prove the results that depend on it.

In fact, the notion of a Ribaucour pair resp transform is more intricate in higher dimensions: in addition to the fact that two submanifolds envelop a congruence of spheres of the right dimension, a generalization of the classical demand that "curvature lines correspond" on the two envelopes needs to be implemented, cf Hertrich-Jeromin (2003, Def 8.2.2) and Burstall and Hertrich-Jeromin (2006, Sect 4):

Definition 3.1 Two immersed submanifolds $x, \hat{x}: \Sigma^{m} \rightarrow S^{n}=\mathbb{P}\left(\mathcal{L}^{n+1}\right)$ in the conformal $n$-sphere, where $\mathcal{L}^{n+1}=\left\{y \in \mathbb{R}^{n+1,1} \mid(y, y)=0\right\}$, form a Ribaucour pair if

(i) they envelop a congruence of $m$-spheres;

(ii) the $(1,1)$-subbundle $x \oplus \hat{x}$ in $\mathbb{R}^{n+1,1}$ is flat. 
Note that Definition 3.1(ii) yields a weak version of the condition that the curvature directions of two hypersurfaces correspond. Also note the slight change of notation: working in the conformal $n$-sphere from the start, $x$ and $\hat{x}$ no longer denote immersions into $\mathbb{R}^{n}$ but into $S^{n}=\mathbb{P}\left(\mathcal{L}^{n+1}\right)$.

Lemma 3.2 Two pointwise distinct immersions $x, \hat{x}: \Sigma^{m} \rightarrow S^{n}=\mathbb{P}\left(\mathcal{L}^{n+1}\right)$

(i) envelop an $m$-sphere congruence if and only if $x^{(1)} \oplus \hat{x}=x \oplus \hat{x}^{(1)}$, where we denote

$$
\left.x^{(1)}\right|_{u}:=x(u) \oplus d_{u} \xi\left(T_{u} \Sigma^{m}\right) \text { for } x=\langle\xi\rangle \text { and } u \in \Sigma^{m}
$$

(ii) form a Ribaucour pair if and only if $d \hat{\xi} \in \Omega^{1}\left(x^{(1)}\right)$ for a suitable lift $\hat{\xi} \in \Gamma(\hat{x})$ of $\hat{x}$.

Lemma 3.2(i) says that the $m$-spheres touching $x$ and containing the points of $\hat{x}$ coincide with those that touch $\hat{x}$ and contain the corresponding points of $x$; Lemma 3.2(ii) implies Lemma 3.2(i) and says that $d \hat{\xi} \perp x$, hence that there is a parallel section $\hat{\xi}$ of $x \oplus \hat{x}$.

At some points it will be useful to consider Legendre lifts into Lie sphere geometry, cf Burstall and Hertrich-Jeromin (2006), however we will mostly stay in Möbius geometry as we aim to keep the notions of points and circles, in particular with a view to the discrete setting, cf Bobenko and Hertrich-Jeromin (2001) or Hertrich-Jeromin (2003, §8.3.16):

Definition 3.3 Two discrete circular nets $x, \hat{x}: \mathbb{Z}^{m} \supset \Sigma^{m} \rightarrow S^{n}=\mathbb{P}\left(\mathcal{L}^{n+1}\right)$ in the conformal $n$-sphere form a Ribaucour pair if corresponding edges are circular, that is, have concircular endpoints.

If two immersed submanifolds $x, \hat{x}: \Sigma^{m} \rightarrow S^{n}$ envelop an $m$-sphere congruence then we obtain a natural identification

$$
x^{(1) \perp} / x \cong\left(x^{(1)} \oplus \hat{x}\right)^{\perp} \cong\left(x \oplus \hat{x}^{(1)}\right)^{\perp} \cong \hat{x}^{(1) \perp} / \hat{x}
$$

of their (weightless) normal bundles, cf Burstall and Hertrich-Jeromin (2006, (4.4)) or Burstall and Calderbank (2010, Sect 8.2): geometrically, this is the identification of Lemma 2.1, where a normal of $x$ is mapped to a normal of $\hat{x}$ by means of a hypersphere that contains the $m$-sphere of the enveloped sphere congruence,

$$
\hat{t}-\frac{(\xi, \hat{t})}{(\xi, \hat{\xi})} \hat{\xi}=s=t-\frac{(\hat{\xi}, t)}{(\hat{\xi}, \xi)} \xi \text { hence }\left\{\begin{array}{l}
\hat{t}+\hat{x}=s+\hat{x} \\
t+x=s+x .
\end{array}\right. \text { and }
$$

Hence we obtain a higher dimensional version of Lemma 2.1:

Lemma 3.4 If two immersed submanifolds $x, \hat{x}: \Sigma^{m} \rightarrow S^{n}$ envelop an $m$-sphere congruence then there is a natural, connection preserving isometric isomorphism (3.2) between their (weightless) normal bundles $x^{(1) \perp} / x$ and $\hat{x}^{(1) \perp} / \hat{x}$. 
A higher dimensional version of the second claim of Corollary 2.3 will be obtained as an immediate consequence, as in the case of space curves: any parallel normal field of one envelope of an $m$-sphere congruence yields one for the other via a "parallel" enveloped hypersphere congruence $s$, hence both envelopes form extended curvature leaves of the envelope of the $m$-parameter family of hyperspheres, in the sense that their tangent spaces are invariant subspaces for the shape operator of the hypersurface, since

$$
0=\nabla^{\perp}(t+x) \Leftrightarrow d(t+x) \in \Omega^{1}\left(x^{(1)} / x\right),
$$

and similarly for the normal field $\hat{t}+\hat{x}$ that corresponds to $t+x$ via (3.2). Conversely, the envelope of a (spacelike) $m$-parameter family of hyperspheres $s: \Sigma^{m} \rightarrow S^{n, 1}$ is foliated by spherical curvature leaves

$$
c(u):=\left(s(u) \oplus d_{u} s\left(T_{u} \Sigma^{m}\right)\right)^{\perp} \cong \mathbb{R}^{n-m, 1} \subset \mathbb{R}^{n+1,1}
$$

of dimension $\operatorname{dim} c(u)-2=n-m-1$; as long as $m<n-1$ any two extended curvature leaves $x$ and $\hat{x}$ complementary to the spherical curvature leaves $c$ are tangent to the $m$-spheres

$$
\left(x \oplus \hat{x} \oplus c^{\perp}\right)(u) \cap s^{\perp}(u)=x(u) \oplus \hat{x}(u) \oplus d_{u} s\left(T_{u} \Sigma^{m}\right) \cong \mathbb{R}^{m+1,1} \subset \mathbb{R}^{n+1,1} .
$$

Note that, in the case $m=n-1$, the spherical "curvature leaves" degenerate to point pairs and we obtain the familiar figure of a hypersphere congruence with its envelope consisting of two sheets.

Summarizing, we obtain the following higher dimensional version of Corollary 2.3:

Corollary 3.5 If $x, \hat{x}: \Sigma^{m} \rightarrow S^{n}$ envelop an m-sphere congruence, and if $x$ has a parallel normal field, then $x$ and $\hat{x}$ yield extended curvature leaves of a hypersurface that is obtained as the envelope of an m-parameter family of hyperspheres.

Conversely, given the envelope of an m-parameter family $s: \Sigma^{m} \rightarrow S^{n, 1}$ of hyperspheres in $S^{n}$, where $m<n-1$, any two extended curvature leaves complementary to its spherical curvature leaves envelop a congruence of $m$-spheres.

This generalization of Corollary 2.3 shows that the construction of a channel surface from a Ribaucour pair of curves only hinges on the enveloped circle congruence and the existence of parallel normal fields, not on the fact that they form a "Ribaucour pair" in the more general sense: if we demand higher dimensional submanifolds $x$ and $\hat{x}$ to form a Ribaucour pair in the sense of Definition 3.1 then more fine structure of $x$ and $\hat{x}$ as extended curvature leaves of a hypersurface can be derived.

Note that the first construction of Corollary 3.5 can be iterated when a sequence of submanifolds that envelop $m$-sphere congruences in a suitable manner is given and if one of the submanifolds admits a parallel normal field: this yields a higher dimensional version of Corollary 2.4.

To get a higher dimensional version of the dimension reduction procedure of Corollary 2.5 we follow the same line of thought as before: given a submanifold $x: \Sigma^{m} \rightarrow S^{n}$ and a fixed hypersphere $e \in S^{n, 1}$, we use a unit normal field 
$t+x \in \Gamma\left(x^{(1) \perp} / x\right)$ and a lift $\xi \in \Gamma(x)$ of $x$ to construct a congruence of hyperspheres

$$
s=t+\frac{1-(e, t)}{(e, \xi)} \xi
$$

that simultaneously touches $x$ and $e$, that is, $s \perp \xi, d \xi$ and $(s, e) \equiv 1$. The touching points

$$
\hat{x}=\langle\hat{\xi}\rangle \text { with } \hat{\xi}:=s-e
$$

of $s$ and $e$ then form a Ribaucour transform of $x$, by Lemma 3.2, as soon as $t+x$ is a parallel normal field of $x$, since

$$
d \hat{\xi}=d s \equiv d t \bmod x^{(1)}
$$

assuming that $\hat{x}$ is complementary to $x$, that is, that $x$ does not intersect the hypersphere $e$, we learn that this condition is also necessary:

Corollary 3.6 A submanifold $x: \Sigma^{m} \rightarrow S^{n}$ admits a Ribaucour transform to a hypersphere $e \in S^{n, 1}$ that it does not meet, $x \not \perp e$, if and only if $x$ admits a parallel normal field $t+x \in \Gamma\left(x^{(1) \perp} / x\right)$; in this case a spherical Ribaucour transform is given by

$$
\hat{x}=\langle s-e\rangle \text { with } s:=t+\frac{1-(e, t)}{(e, \xi)} \xi \text { and } \xi \in \Gamma(x) .
$$

Note that, in this dimension reduction construction, $x$ and $\hat{x}$ form a Ribaucour pair as soon as they envelop an $m$-sphere congruence: since $(d s, \xi)=-(s, d \xi)=0$ we conclude that

$$
d s \in \Omega^{1}\left(x^{(1)} \oplus \hat{x}\right) \Leftrightarrow d s \in \Omega^{1}\left(x^{(1)}\right) .
$$

This observation is confirmed by the discrete case. Given a circular net $x: \mathbb{Z}^{m} \supset$ $\Sigma^{m} \rightarrow S^{n}$, and a hypersphere $e \in S^{n, 1}$ that the net does not meet we construct a spherical net iteratively, by evolving along edges using the intersection of the fixed sphere with the circumcircle of an edge and an initial point for the edge, as in the 1-dimensional case Theorem 2.6. This construction is consistent, and yields a circular net, by the Möbius geometric formulation of Miguel's theorem: given seven vertices of a cube with circular faces, the eighth vertex can be constructed uniquely. Namely, considering a face of the original net $x$ and an initial point of the corresponding face of $\hat{x}$ the construction of the face of $\hat{x}$ is completed on the 2-sphere containing the five starting points, cf Bobenko and Hertrich-Jeromin (2001, Sect 4). A standard dimension count then shows that corresponding $m$-cells of the circular nets $x$ and $\hat{x}$ lie on $m$-spheres:

Corollary 3.7 Any circular net $x: \mathbb{Z}^{m} \supset \Sigma^{m} \rightarrow S^{n}$ admits a Ribaucour transform to a fixed hypersphere $e \in S^{n, 1}$ that it does not meet, $x \not \perp e$; the two nets envelop 
a discrete $m$-sphere congruence, in the sense that corresponding $m$-cells lie on $m$ spheres.

As in the case of curves in Corollary 2.7, the dimension reductions of Corollary 3.6 resp Corollary 3.7 can be used to introduce Ribaucour coordinates for a submanifold with flat normal bundle-where "enough" spherical Ribaucour transforms exist, cf Dajczer et al. (2007, Thm 1).

Thus suppose that $t_{i}+x \in \Gamma\left(x^{(1) \perp} / x\right), i=1, \ldots, n-m$, are parallel unit normal fields of an immersed submanifold $x: \Sigma^{m} \rightarrow S^{n}$ and let $e_{1}, \ldots, e_{n-m} \in S^{n, 1}$ denote an orthonormal system that defines an $m$-sphere in the conformal $n$-sphere via

$$
e=\left\langle e_{i} \mid i=1, \ldots, m\right\rangle^{\perp} \cong \mathbb{R}^{m+1,1} \subset \mathbb{R}^{n+1,1}
$$

Now we let $\xi \in \Gamma(x)$ and iterate (3.3) to obtain an iterated Ribaucour transform of $x$ into the $m$-sphere $e$ : with a first Ribaucour transformation of $x$ into the $i$ th hypersphere $e_{i}$ and its parallel normal field obtained by (3.2) from the $j$ th parallel normal field of $x$,

$$
\xi_{i}=\left(t_{i}-e_{i}\right)+\frac{1-\left(e_{i}, t_{i}\right)}{\left(e_{i}, \xi\right)} \xi \text { and } t_{i j}-\frac{\left(\xi, t_{i j}\right)}{\left(\xi, \xi_{i}\right)} \xi_{i}=t_{j}-\frac{\left(\xi_{i}, t_{j}\right)}{\left(\xi, \xi_{i}\right)} \xi
$$

a lengthy but straightforward computation reveals that

$$
\begin{aligned}
\xi_{i j}= & \left(t_{j}-e_{j}\right)+\frac{\left(1-\left(e_{j}, t_{j}\right)\right)\left(e_{i}, \xi\right)+\left(e_{i}, t_{j}\right)\left(e_{j}, \xi\right)}{\left(1-\left(e_{i}, t_{i}\right)\right)\left(e_{j}, \xi\right)+\left(e_{j}, t_{i}\right)\left(e_{i}, \xi\right)}\left(t_{i}-e_{i}\right) \\
& +\frac{\left(1-\left(e_{i}, t_{i}\right)\right)\left(1-\left(e_{j}, t_{j}\right)\right)-\left(e_{i}, t_{j}\right)\left(e_{j}, t_{i}\right)}{\left(1-\left(e_{i}, t_{i}\right)\right)\left(e_{j}, \xi\right)+\left(e_{j}, t_{i}\right)\left(e_{i}, \xi\right)} \xi
\end{aligned}
$$

we observe that, up to scaling, $\xi_{i j}$ is symmetric in $i$ and $j$, thus confirming Bianchi's permutability theorem for the particular Ribaucour transformations we use, cf Burstall and Hertrich-Jeromin (2006, Sect 3) or Dajczer et al. (2007, Cor 16). Once the circularity claim of Bianchi's permutability theorem is established for $x, x_{i}, x_{j}$ and $x_{i j}$,

$$
x_{i j}=\left\langle\xi_{i j}\right\rangle \quad \text { with } \xi_{i j} \in \Gamma\left(x \oplus x_{i} \oplus x_{j}\right)
$$

we obtain an alternative approach to determining $x_{i j}$ : the conditions $\xi_{i j} \perp e_{i}, e_{j}$ for $x_{i j}$ lead to a homogeneous system of linear equations for the coefficients in (3.4),

$$
\begin{aligned}
\xi_{i j}= & a_{0} \xi+a_{i}\left(t_{i}-e_{i}\right)+a_{j}\left(t_{j}-e_{j}\right) \text { with } \\
& \left\{\begin{array}{l}
0=\left(e_{i}, \xi\right) a_{0}-\left(1-\left(e_{i}, t_{i}\right)\right) a_{i}+\left(e_{i}, t_{j}\right) a_{j}, \\
0=\left(e_{j}, \xi\right) a_{0}+\left(e_{j}, t_{i}\right) a_{i}-\left(1-\left(e_{j}, t_{j}\right)\right) a_{j} ;
\end{array}\right.
\end{aligned}
$$

Cramer's rule then recovers the coefficients of (3.4) up to scaling-as long as the equations are independent, which can be achieved by ensuring that $x$ does not hit the hyperspheres $e_{i}^{\perp}$ and $e_{j}^{\perp}$, and $x_{i}$ and $x_{j}$ do not hit the target $(n-2)$-sphere $\left\langle e_{i}, e_{j}\right\rangle^{\perp}$ 
of $x_{i j}$ : we require that the dimension reduction of Corollary 3.6 works in every step of the iteration.

Using (3.5) it is now straightforward to formulate the general case: as every permutation of a finite set is a composition of transpositions our dimension reduction is independent of order, and circularity of Bianchi quadrilaterals ensures that (3.5) generalizes. More precisely,

Theorem and Definition 3.8 Suppose that $t_{i}+x \in \Gamma\left(x^{(1) \perp} / x\right), i=1, \ldots, n-m$, are parallel unit normal fields of an immersed submanifold $x: \Sigma^{m} \rightarrow S^{n}$ and let $e=\left\langle e_{i} \mid i=1, \ldots, m\right\rangle^{\perp}$ denote an $m$-sphere, given in terms of orthogonally intersecting hyperspheres $e_{1}, \ldots, e_{n-m} \in S^{n, 1}$. Then $\hat{\xi}$ of the form

$$
\hat{\xi}:=a_{0} \xi+\sum_{i=1}^{n-m} a_{i}\left(t_{i}-e_{i}\right) \neq 0, \operatorname{solving}\left(\hat{\xi}, e_{i}\right)=0 \text { for } i=1, \ldots, n-m,
$$

defines an $(n-m)$-fold Ribaucour transform $\hat{x}=\langle\hat{\xi}\rangle: \Sigma^{m} \rightarrow S^{n}$ of $x$ into the $m$-sphere $e$.

We say that $\hat{x}$ defines Ribaucour coordinates for the submanifold $x$.

Note that the choices of (parallel resp constant) normal fields for $x$ and the $m$-sphere $e$ establishes an isometric and connection preserving isomorphism between normal bundles: this isomorphism is the source of our vectorial Ribaucour transformation that establishes the Ribaucour coordinates, cf Dajczer et al. (2007, Thm 1).

Based on Corollary 3.7 Ribaucour coordinates for discrete principal nets can be introduced in a similar way: in order to ensure that permutability holds we need to choose initial points for the spherical Ribaucour transforms suitably-Miguel's theorem then guarantees that the double Ribaucour reduction $x_{i j}$ is independent of order and corresponding points of the circular nets $x, x_{i}, x_{j}$ and $x_{i j}$ are concircular, see Hertrich-Jeromin (2003, Sect 8.5). Thus in the case of an $m$-dimensional net in $S^{n}$ initial points need to be suitably chosen on an $(n-m-1)$-sphere containing the initial point of $x$ and the corresponding point of the target net defining the Ribaucour coordinates.

Theorem and Definition 3.9 Suppose that $x: \mathbb{Z}^{m} \supset \Sigma^{m} \rightarrow S^{n}$ is a circular net and fix an $m$-sphere $e=\left\langle e_{i} \mid i=1, \ldots, m\right\rangle^{\perp}$ in terms of orthogonally intersecting hyperspheres $e_{1}, \ldots, e_{n-m} \in S^{n, 1}$; further fix $i_{0} \in \Sigma^{m}$ and choose an $(n-m)$-cube

$$
\{0,1\}^{n-m} \ni \varepsilon \mapsto y_{\varepsilon} \in S^{n} \text { with } \begin{cases}y_{\varepsilon}=x\left(i_{0}\right) & \text { for } \varepsilon=0 \text { and } \\ y_{\varepsilon} \in\left\langle\varepsilon_{1} e_{1}, \ldots, \varepsilon_{n-m} e_{n-m}\right\rangle^{\perp} & \text { for } \varepsilon \neq 0\end{cases}
$$

and with circular faces. Then the $(n-m)$-fold iterated Ribaucour reduction with initial points $y_{\varepsilon}$

$$
\hat{x}: \Sigma^{m} \rightarrow e=\left\langle e_{1}, \ldots, e_{n-m}\right\rangle^{\perp} \subset S^{n}
$$

and is independent of the order of transformations. 
We say that $\hat{x}$ defines Ribaucour coordinates for the discrete circular net $x$.

While the dimension reduction via Ribaucour transformations works perfectly in higher dimensions, the interpolation by Ribaucour transformations of Theorem 2.8 hinges on the flatness of the Demoulin vector bundle (2.10) - which is no longer trivially satisfied in higher dimensions. Indeed, in Burstall and Hertrich-Jeromin (2006, Sect 6) we provide an example of two surfaces that have one common (totally umbilic) Ribaucour transform but not more, so that permutability fails: in particular, the curvature lines on the common Ribaucour transform (obtained from the respective Ribaucour transformation) do not line up.

This situation is typical: by introducing Ribaucour coordinates of Definition 3.8 for two $m$-dimensional submanifolds with flat normal bundle in $S^{n}$, the two submanifolds may be (locally) obtained by a sequence of $2(n-m)$ Ribaucour transformations from each other, however, their curvature directions will in general not line up in the desired way.

In the discrete case, where curvature line coordinates are inherent, we learn from these observations that interpolation by Ribaucour transformations fails in general: a simple discretization of the Dupin cyclides used in Burstall and Hertrich-Jeromin (2006, Sect 6), by sampling curvature lines, provides counter-examples.

Acknowledgements Open access funding provided by TU Wien (TUW). This work would not have been possible without the valuable and enjoyable discussions with A. Fuchs, C. Müller, M. Pember, F. Rist and G. Szewieczek about the subject. Furthermore, we gratefully acknowledge financial support of the second author by the Bath Institute for Mathematical Innovation; and of the third author by the TU Wien Doctoral Training Centre "Computational Design".

Open Access This article is distributed under the terms of the Creative Commons Attribution 4.0 International License (http://creativecommons.org/licenses/by/4.0/), which permits unrestricted use, distribution, and reproduction in any medium, provided you give appropriate credit to the original author(s) and the source, provide a link to the Creative Commons license, and indicate if changes were made.

\section{References}

Blaschke, W.: Vorlesungen über Differentialgeometrie III. Grundlehren, Vol. XXIX. Springer, Berlin (1929) Bo, P., Kilian, M., Pottmann, H., Wallner, J., Wang, W.: Circular arc structures. ACM Trans. Graph. Proc. SIGGRAPH 30, 101 (2011)

Bobenko, A., Hertrich-Jeromin, U.: Orthogonal nets and Clifford algebras. Tôhoku Math. Publ. 20, 7-22 (2001)

Bobenko, A., Huhnen-Venedy, E.: Curvature line parametrized surfaces and orthogonal coordinate systems: discretization with Dupin cyclides. Geom. Dedicata 159, 207-237 (2012)

Burstall, F., Calderbank, D.: Conformal submanifold geometry I-III (2010). EPrint arXiv:1006.5700

Burstall, F., Hertrich-Jeromin, U.: The Ribaucour transformation in Lie sphere geometry. Differ. Geom. Appl. 24, 503-520 (2006)

Burstall, F., Hertrich-Jeromin, U., Müller, C., Rossman, W.: Semi-discrete isothermic surfaces. Geom. Dedicata 183, 43-58 (2016)

Corro, A., Tenenblat, K.: Ribaucour transformations revisited. Commun. Anal. Geom. 12, 1055-1082 (2004)

Dajczer, M., Florit, L., Tojeiro, R.: The vectorial Ribaucour transformation for submanifolds and its applications. Trans. Am. Math. Soc. 359, 4977-4997 (2007)

de Lima, L., Roitman, P.: Constant mean curvature one surfaces in hyperbolic 3-space using the BianchiCalò method. Ann. Braz. Acad. Sci. 74, 19-24 (2002)

Hertrich-Jeromin, U., Rossman, W., Szewieczek, G.: Discrete channel surfaces (in preparation) 
Hertrich-Jeromin, U.: Introduction to Möbius differential geometry. London Mathematical Society Lecture Note Series, Vol. 300. Cambridge University Press, Cambridge (2003)

Müller, C., Wallner, J.: Semi-discrete isothermic surfaces. Res. Math. 63, 1395-1407 (2013)

Pember, M., Szewieczek, G.: Channel surfaces in Lie sphere geometry (2017). EPrint arXiv:1709.02224 